\title{
AUTORREPORTE DE ACCIDENTES DE TRÁNSITO EN UNA ENCUESTA NACIONAL EN LA POBLACIÓN URBANA DE PERÚ
}

\author{
Paolo Wong ${ }^{1, a, b}$, César Gutiérrez ${ }^{1, a, c}$, Franco Romaníi,a,d
}

\begin{abstract}
RESUMEN
Objetivos. Estimar la frecuencia del autorreporte de accidente de tránsito como antecedente en el último año en la población general y determinar qué factores se encuentran asociados. Materiales y métodos. Se realizó un análisis secundario de los datos de la III Encuesta Nacional de Consumo de Drogas en la Población General del Perú 2006 de DEVIDA. Se midieron las variables sociodemográficas: edad, sexo, región de procedencia, nivel educativo alcanzado y estado civil. Asimismo, se evaluó el consumo de drogas legales, ilegales y médicas. La variable de respuesta fue el autorreporte de accidente de tránsito. Se realizó análisis descriptivo y bivariado de las variables sociodemográficas y de consumo de drogas (legales e ilegales) con el autorreporte de accidente de tránsito. Resultados. La frecuencia de reporte de accidente de tránsito en el último año según la encuesta fue de 2,93\% (IC95\%: 2,92-2,94). Los factores asociados a autorreportar un accidente de tránsito son: vivir en la selva (OR: 2,03; IC95\%: 1,55-2,65), género masculino (OR: 1,79; IC95\%: 1,46-2,22), consumo de drogas legales en el último año (OR: 1,98; IC95\%: 1,53-2,55),consumo de alcohol en el último año (OR: 1,82; IC95\%: 1,44-2,32) y consumo de drogas médicas en el último año (OR: 2,45; IC95\%: 1,63-3,68). Conclusiones. La prevalencia de autorreporte de accidente de tránsito en el último año fue muy elevada comparada con estudios similares y otras fuentes reportantes. Las variables asociadas con el antecedente de accidente de tránsito son: el vivir en la selva, ser varón, el consumo de drogas legales en el último año, especialmente alcohol y consumo de drogas médicas en el último año. Es necesario reflexionar acerca del sistema de información sobre accidentes de tránsito a fin de una mayor caracterización del problema haciendo énfasis en el consumo de drogas legales.
\end{abstract}

Palabras clave: Accidentes de tránsito; Riesgo; Abuso de alcohol; Muestreo por conglomerados; Fuentes de datos; Perú (fuente: DeCS BIREME).

\section{SELF-REPORTING OF ROAD TRAFFIC ACCIDENTS IN A NATIONAL SURVEY OF URBAN POPULATION IN PERU}

\begin{abstract}
Objectives. To estimate the frequency of self-reporting of road traffic accidents in the previous year in the general population and to determine the associated factors. Materials and methods. We conducted a secondary analysis of the data of the III National Survey of Drug Use in the General Population of Peru, 2006. We measured socio-demographical variables: age, gender, place of origin, educational level and marital status. We also evaluated the use of legal, illegal and medical drugs. The independent variable was the self-reporting of a road traffic accident. We performed the descriptive, bivariate and multivariate analysis of the socio-demographical variables and the drug use (legal and illegal), together with the self-reporting of the traffic accident. Results. The frequency of reporting of road traffic accidents in the last year according to the survey was $2.93 \%(95 \% \mathrm{Cl}: 2.92-2.94)$. The associated factors for self-reporting of a road traffic accident were: to live in the jungle areas (OR: $2.03 ; 95 \% \mathrm{Cl}: 1.55-2.65)$, male gender (OR: $1.79 ; 95 \% \mathrm{Cl}: 1.46-2.22)$, legal drugs use in the last year (OR: $1.98,95 \% \mathrm{Cl}: 1.53-2.55)$, alcohol consumption in the last year (OR: 1.82; 95\% Cl: 1.44-2.32) and medical drugs use in the last year (OR: $2,45,95 \% \mathrm{Cl} 1,63-3,68)$. Conclusions. The prevalence of self-reporting of road traffic accidents in the last year was very high compared to similar studies and other reporting sources. The variables associated with having had a traffic accident were: living in the jungle area, being male, legal drug use in the last month, especially alcohol and medical drug use in the last month. It is necessary to think carefully about the information system of the road traffic accidents in order to achieve a better picture of the problem putting emphasis in the legal drugs use.
\end{abstract}

Key words: Accidents, traffic; Risk; Alcohol abuse; Cluster sampling; Data sources; Peru (source: MeSH NLM).

\section{INTRODUCCIÓN}

El impacto actual que tienen los accidentes de tránsito en la mortalidad y morbilidad de la población es de gran magnitud. Cada año se producen en el mundo miles de choques, atropellos, volcaduras, despistes o caídas desde vehículos, que conllevan a muertes prematuras, lesiones permanentes To temporales $y$ desórdenes psicológicos o emocionales que tienen serias implicaciones sanitarias, sociales y económicas $(1,2)$. Esa realidad, en términos personales y colectivos, no nos es ajena. Podríamos decir que todos, en determinado momento, hemos presenciado algún tipo de accidente en nuestras vías $y$, diariamente, los medios

1 Sección Epidemiología, Instituto de Medicina Tropical, Facultad de Medicina, Universidad Nacional Mayor de San Marcos, Lima, Perú.

a Médico Cirujano; ${ }^{\mathrm{b}}$ Maestrando en Bioquímica; ${ }^{\mathrm{c}}$ Magíster en Epidemiología; ${ }^{\mathrm{d}}$ Maestrando en Ingeniería Biomédica. 
de comunicación nos convierten en testigos de muchos otros.

La Organización Mundial de la Salud (OMS) informa que alrededor de 1,2 millones de personas mueren en el mundo cada año debido a un accidente de tránsito y cerca de 50 millones resultan lesionados. (2) Según el Banco Mundial, para el año 2030, los accidentes de tránsito ocuparán el tercer lugar en el ranking de carga por lesiones a nivel global (según los años de vida ajustados en función de la discapacidad, AVAD), sólo por debajo de la enfermedad isquémica de miocardio y la depresión mayor unipolar, superando a la enfermedad cerebro-vascular, enfermedad pulmonar obstructiva crónica (EPOC), tuberculosis, Síndrome de Inmunodeficiencia Adquirida (SIDA) e inclusive a las guerras ${ }^{(2)}$. En cuanto al orden relativo de las diez causas principales de muerte en el mundo, para el mismo año, los accidentes de tránsito ocuparán el octavo lugar, luego de la cardiopatía isquémica, los trastornos cerebro-vasculares, SIDA, EPOC, infecciones de las vías respiratorias inferiores, diabetes mellitus y los cánceres de la tráquea, bronquios y pulmones ${ }^{(2)}$.

Asimismo, la OMS señala que las pérdidas humanas -la peor consecuencia de cualquier fenómeno desde todo punto de vista- y las incapacidades resultantes no son los únicos factores de impacto negativo de los accidentes de tránsito en la población: por cada persona que fallece debido a esta causa, 30 son hospitalizadas y 300 son atendidas en los servicios de emergencias; es decir, la gran carga que este fenómeno impone al sistema de salud un consumo importante de recursos económicos, materiales y humanos ${ }^{(2,3)}$.

En Perú, debido al crecimiento desordenado del parque automotor, a un sistema de educación vial ineficiente y a otros factores, el riesgo de ocurrencia de accidentes de tránsito ha aumentado considerablemente dando lugar a un grave problema de salud pública. En 2004, el Ministerio de Salud de Perú (MINSA) concluyó que la tasa de mortalidad por éstos es una de las más altas de Latinoamérica (30 personas fallecidas por cada $10 \mathrm{mil}$ vehículos) ${ }^{(4)}$. El Plan Nacional de la Estrategia Sanitaria Nacional de Accidentes de Tránsito del MINSA informa que durante el año 2007, la mortalidad por accidentes de tránsito en el Perú llegó a 3510 fallecidos y casi 50 mil lesionados. Esta cantidad de heridos se ha incrementado en un $5,88 \%$ con respecto al año $2006^{(5)}$.

Según los datos que brinda la Policía Nacional del Perú (PNP), cada 24 horas mueren diez peruanos debido a accidentes de tránsito y en 2007 la tasa de eventos negativos de tránsito - es decir, con víctimas mortales - llegó a 12,72 por cada 100 mil habitantes. Ese año, la PNP registró 79972 accidentes de tránsito, con lo que la tasa de eventos llegaría a 289,8 por cada 100 mil habitantes ${ }^{(5)}$. Un reciente informe de la Defensoría del Pueblo sobre el transporte terrestre de pasajeros indica que durante el año 2009 murieron aproximadamente 3243 personas, mientras que 48395 resultaron heridas a consecuencia de los accidentes de tránsito en todo el Perú ${ }^{(6)}$. Sólo en los últimos cuatro años, los accidentes de tránsito han dejado cerca de 120 mil discapacitados ${ }^{(5-7)}$.

En el informe de carga de enfermedad y lesiones en el Perú, se indica que los accidentes de tránsito representan la primera causa de carga de enfermedad en el país con el mayor número de años de vida saludable perdidos (AVISA) ${ }^{\left({ }^{8}\right)}$. Según cálculos del MINSA, el costo de los daños producidos por accidentes de tránsito en el Perú podría ascender a mil millones de dólares por año (de 1,5 a $2 \%$ del Producto Bruto Interno) ${ }^{(5)}$.

Informes oficiales e internacionales así como muchas investigaciones científicas indican que el tipo de accidente más común es el choque; que la razón entre hombres y mujeres es de dos a uno; asimismo, que el grupo de edad más comprometido es el adulto joven, además, que el trastorno más común a rehabilitar es la lesión medular (5,9-11). También señalan que la causa principal de los accidentes está en manos del conductor del vehículo (por exceso de velocidad, por imprudencia o negligencia) $)^{(5,9,10)}$.

Se ha documentado que, además de los factores descritos en el párrafo previo, el uso de drogas legales -sobre todo el alcohol- está asociado con mayor cantidad de accidentes de tránsito, con mayor mortalidad, con mayor gravedad de las lesiones ocasionadas y al pobre pronóstico de los supervivientes ${ }^{(11,12)}$. Por ello, investigadores y autoridades coinciden en lo esencial que es disponer de información precisa acerca de la epidemiología de los accidentes de tránsito para decidir el orden de prioridad de los problemas de salud pública, vigilar las tendencias y evaluar los programas de intervención ${ }^{(9,10,13)}$.

Se entiende que una estrategia importante para poder construir dicho sistema de información es obtener los datos de la mayor cantidad de fuentes disponibles para poder lograr la mejor estimación de la situación. Sin embargo, la mayoría de las fuentes utilizadas en los estudios sobre accidentes son registros estadísticos de las instituciones involucradas en la atención de éstos (estadística policial, aseguradoras, hospitales, morgue). Pocos indagan en el autorreporte de antecedentes de accidentes de tránsito en cada individuo, datos que pueden no ser recopilados en su totalidad por las fuentes oficiales ya descritas, sobre todo en lo concerniente a accidentes leves o de consecuencia no fatal. Las 
encuestas en población general, a veces objetivadas en otros fines, pueden aportar datos sobre supervivientes a accidentes de tránsito que, al analizar junto con otras cifras, adquieren singular relevancia ${ }^{(13)}$.

La III Encuesta Nacional de Consumo de Drogas en la Población General del Perú 2006 a cargo de la Comisión Nacional para el Desarrollo y Vida Sin Drogas (DEVIDA) se propuso conocer la magnitud y factores asociados con el consumo de drogas en el país ${ }^{(14)}$. Bajo ese objetivo, se estudió a través de una encuesta de hogares, a cerca de 12 mil personas, recopilando -además de información general y de consumo de drogas-datos sobre integración familiar, vulnerabilidad e inclusión social así como información sobre salud y estilo de vida. En este último acápite, el estudio recogió datos acerca del antecedente de haber sufrido algún accidente de tránsito en el último año, por lo que este estudio se centra en dicha pregunta. Se describe la frecuencia del autorreporte de accidente de tránsito en el último año en la población general y se evalúa la asociación a algunas características específicas. De igual manera, se reflexiona acerca de los diferentes indicadores, fuentes y formas de evaluar la magnitud de este importante problema de salud pública.

\section{MATERIALES Y MÉTODOS}

\section{FUENTE DE INFORMACIÓN}

La III Encuesta Nacional de Consumo de Drogas en la Población General del Perú 2006 de DEVIDA entrevistó a 11825 personas de 12 a 64 años de edad en hogares en zonas urbanas de 43 ciudades del país. El muestreo de la encuesta fue probabilístico, multietápico y por conglomerados. Dicha muestra es representativa de la población total del Perú. El instrumento constó de 156 preguntas dentro de las cuales se interrogó -en el capítulo sobre Salud y Estilo de Vida- acerca del antecedente de haber sufrido algún accidente de tránsito en el último año (pregunta 38.2).

El informe de la encuesta se encuentra disponible en la página Web de DEVIDA (www.devida.gob.pe/ Documentacion/Encuesta_Nacional_De_Consumo_ De_Drogas.pdf). Se contó con la autorización escrita de los realizadores de la encuesta para la utilización de los datos, los cuales no incluyen la identificación de las personas encuestadas, por lo que no fue necesario solicitar el consentimiento informado.

\section{DEFINICIÓN DE VARIABLES}

Definimos autorreporte como la respuesta positiva que realizó cada individuo tras la pregunta “ ¿Ha tenido usted algún accidente de tránsito en el último año?" (Pregunta 38.2); lo que no necesariamente implica un reporte policial u hospitalario. Se utilizó la base de datos de dicha encuesta, analizando el reporte de accidente de tránsito en el último año de acuerdo con la edad y sexo de los encuestados, el área geográfica de donde proceden (costa, sierra y selva), el nivel educativo alcanzado (ninguno, inicial, primario, secundario, superior) y el estado civil (clasificando como "unido" a los convivientes o casados y como "no unido" a los solteros, separados, viudos o divorciados). Se utilizaron los factores de expansión de la encuesta. Se realizó un mapa de acuerdo con la prevalencia reportada por región política del Perú dividiéndolas en tres niveles de acuerdo con su magnitud.

Asimismo, se examinó y analizó el antecedente de consumo de drogas en el último año (drogas legales: alcohol, tabaco; drogas ilegales: cocaína, marihuana, $\mathrm{PBC}$, éxtasis, crack, etc. y drogas médicas) con respecto al reporte de accidente de tránsito en el último año. Se analizó también el consumo de alcohol en el último año de manera independiente de las otras drogas legales.

\section{ANÁLISIS ESTADÍSTICO}

Se describieron las frecuencias absolutas y relativas, estimándose parámetros poblacionales, considerando los factores de expansión propios de la encuesta. Posteriormente, se realizó el análisis bivariado de los otros factores estudiados mediante la prueba de Ji-cuadrado en el caso de dos variables categóricas y la prueba $t$ de Student cuando se compararon medias, en ambos casos se evaluaron los supuestos correspondientes; asimismo se calculó el Odds Ratio (OR). Para todas las pruebas se usó un nivel de significancia de 0,05, concordante con lo usado en el proceso de muestreo. Se utilizaron los programas estadístico Statistical Packcage for Social Sciences SPSS ${ }^{\circledR}$ (Chicago, Illinois) versión 16.0 para Windows y Epi-Dat尺 (OPS, Washington DC) versión 3.1 para Windows.

\section{RESULTADOS}

\section{CARACTERÍSTICAS GENERALES DE LA POBLACIÓN ENCUESTADA}

El estudio incluyó 11825 personas, que representan a 10754650 habitantes (muestra expandida). La media de edad fue de $34,3 \pm 14,4$ años. El $56,6 \%$ fue del sexo femenino. Respecto al estado civil, el 49,0\% estuvieron unidos (casados o convivientes). En cuanto al nivel educativo alcanzado, el $0,5 \%$ no tuvo estudios, $0,3 \%$ alcanzó el nivel inicial, 9,9\% alcanzó el nivel primario, $49,7 \%$ alcanzó el nivel secundario y el 39,4\% alcanzó el 
nivel superior. Por último, el $76,4 \%$ reside en la región costa, el $17,0 \%$ reside en la sierra y el $6,6 \%$ es de la selva.

\section{AUTORREPORTE DE ACCIDENTE DE TRÁNSITO}

La frecuencia de autorreporte de accidente de tránsito en el último año según la encuesta fue de 2,93\% (IC95\% 2,92-2,94); es decir, 29 accidentes de tránsito autorreportados (ATR) por cada 1000 habitantes.

\section{ASPECTOS SOCIODEMOGRÁFICOS Y ATR}

De las personas que presentaron ATR, el 59,7\% (187 988) de sexo masculino y 40,3\% (127 005) del femenino. Existe asociación entre el antecedente de accidente y el sexo masculino ( $p<0.001$, OR: 1,79; IC95\% 1,46-2,22).
La media de la edad de las personas que respondieron haber tenido un accidente de tránsito en el último año fue de 34,1 $\pm 13,2$ años, siendo similar a la edad de quienes no reportaron haber tenido un accidente de tránsito el último año $(34,3 \pm 14,3)(p=0,793, t$ de Student). Las cifras sobre las características de la población se observan en la Tabla 1.

En relación al grado de instrucción y el autorreporte de accidente de tránsito el último año, las personas con menor y mayor grado de instrucción fueron las que reportaron la mayor proporción $(3,6 \%)$, siendo estadísticamente significativa la relación entre grado de instrucción y el autorreporte de accidente de tránsito $(p=0,030)$. En el caso del estado civil, la proporción de reporte de accidentes entre personas unidas y no unidas fue similar (3,0\% en cada grupo, $p=0,963)$.

Tabla 1. Características de acuerdo con el antecedente de accidente de tránsito en el último año, Perú 2006.

\begin{tabular}{|c|c|c|c|c|c|c|c|}
\hline \multirow{3}{*}{ Característica } & \multirow{3}{*}{$\begin{array}{c}\text { Total } \\
\mathbf{n}\end{array}$} & \multicolumn{4}{|c|}{ Autorreporte de accidente de tránsito } & \multirow{3}{*}{ OR (IC 95\%) } & \multirow{3}{*}{$p$ valor } \\
\hline & & \multicolumn{2}{|c|}{ Sí } & \multicolumn{2}{|c|}{ No } & & \\
\hline & & $\mathbf{n}$ & $\%$ & $\mathbf{n}$ & $\%$ & & \\
\hline \multicolumn{8}{|l|}{ Edad } \\
\hline Media \pm DS & & \multicolumn{2}{|c|}{$34,09 \pm 13,2$} & \multicolumn{2}{|c|}{$34,27 \pm 14,3$} & & $0,793^{*}$ \\
\hline 12 a 19 años & 2161 & 51 & 2,4 & 2110 & 97,6 & 1 & $0,132 \dagger$ \\
\hline 20 a 29 años & 2763 & 93 & 3,4 & 2670 & 96,6 & $1,44(1,02-2,04)$ & \\
\hline 30 a 39 años & 2762 & 94 & 3,4 & 2668 & 96,6 & $1,46(1,03-2,06)$ & \\
\hline 40 a 49 años & 2020 & 63 & 3,1 & 1957 & 96,9 & $1,33(0,92-1,94)$ & \\
\hline 50 años o más & 2115 & 55 & 2,6 & 2060 & 97,4 & $1,10(0,75-1,62)$ & \\
\hline \multicolumn{8}{|l|}{ Región } \\
\hline Costa & 7135 & 181 & 2,5 & 6954 & 97,5 & 1 & $<0,001 \dagger$ \\
\hline Sierra & 3090 & 95 & 3,1 & 2995 & 96,9 & $1,22(0,95-1,57)$ & \\
\hline Selva & 1596 & 80 & 5,0 & 1516 & 95,0 & $2,03(1,55-2,65)$ & \\
\hline \multicolumn{8}{|l|}{ Nivel de estudios } \\
\hline No tiene, no sabe, Inicial & 137 & 5 & 3,6 & 132 & 96,4 & 1 & $0,030 \dagger$ \\
\hline Primaria & 1388 & 39 & 2,8 & 1349 & 97,2 & $0,76(0,30-1,97)$ & \\
\hline Secundaria & 5617 & 145 & 2,6 & 5472 & 97,4 & $0,70(0,28-1,73)$ & \\
\hline Superior & 4679 & 167 & 3,6 & 4512 & 96,4 & $0,98(0,39-2,42)$ & \\
\hline \multicolumn{8}{|l|}{ Estado Civilł } \\
\hline Unido & 5896 & 178 & 3,0 & 5718 & 97,0 & 1 & $0,963 \dagger$ \\
\hline No unido & 5925 & 178 & 3,0 & 5747 & 97,0 & $1,01(0,81-1,24)$ & \\
\hline \multicolumn{8}{|l|}{ Drogas legales no médicas " } \\
\hline Sí & 7563 & 276 & 3,6 & 7287 & 96,4 & $1,98(1,53-2,55)$ & $<0,001 \dagger$ \\
\hline No & 4150 & 78 & 1,9 & 4072 & 98,1 & 1 & \\
\hline \multicolumn{8}{|l|}{ Drogas médicas" } \\
\hline Sí & 397 & 27 & 6,8 & 370 & 93,2 & $2,45(1,63-3,68)$ & $<0,001 \dagger$ \\
\hline No & 11309 & 327 & 2,9 & 10982 & 97,1 & 1 & \\
\hline \multicolumn{8}{|l|}{ Drogas ilegales" } \\
\hline Sí & 96 & 6 & 6,3 & 90 & 93,7 & $2,17(0,94-4,99)$ & $0,062 \dagger$ \\
\hline No & 11573 & 345 & 3,0 & 11228 & 97,0 & 1 & \\
\hline \multicolumn{8}{|l|}{ Alcohol" } \\
\hline Sí & 7111 & 260 & 3,7 & 6851 & 96,3 & $1,82(1,44-2,32)$ & $<0,001 \dagger$ \\
\hline No & 4614 & 94 & 2,0 & 4520 & 98,0 & 1 & \\
\hline
\end{tabular}

DS: Desviación estándar; * Prueba $t$ de Student; † Prueba de Ji-cuadrado;

‡ Unido: casado o conviviente; No unido: soltero, separado, viudo o divorciado

I Consumo en el último año. 
Tabla 2. Porcentaje de autorreporte de accidente de tránsito en el último año según departamento. Perú, 2006.

\begin{tabular}{|c|c|c|c|c|c|c|}
\hline \multirow{3}{*}{ Región } & \multirow{3}{*}{$\begin{array}{c}\text { Muestra } \\
n^{*}\end{array}$} & \multirow{3}{*}{$\begin{array}{l}\text { Población } \\
\mathbf{N}^{* *}\end{array}$} & \multicolumn{4}{|c|}{ Autorreporte de accidente de tránsito } \\
\hline & & & \multicolumn{2}{|c|}{ Sí } & \multicolumn{2}{|c|}{ No } \\
\hline & & & $\mathbf{N}^{* *}$ & $\%$ & $\mathbf{N}^{* *}$ & $\%$ \\
\hline Ucayali & 240 & 166890 & 13212 & 7,9 & 153678 & 92,1 \\
\hline Ayacucho & 229 & 176929 & 11425 & 6,5 & 165504 & 93,5 \\
\hline Cajamarca & 341 & 189554 & 11165 & 5,9 & 178389 & 94,1 \\
\hline Huánuco & 330 & 182818 & 10064 & 5,5 & 172754 & 94,5 \\
\hline Puno & 397 & 301131 & 16074 & 5,3 & 285057 & 94,7 \\
\hline Tacna & 192 & 167706 & 8735 & 5,2 & 158971 & 94,8 \\
\hline Apurímac & 306 & 76751 & 3527 & 4,6 & 73224 & 95,4 \\
\hline Madre de Dios & 158 & 47099 & 2087 & 4,4 & 45012 & 95,6 \\
\hline Ica & 342 & 452946 & 19075 & 4,2 & 433871 & 95,8 \\
\hline San Martín & 210 & 146029 & 5563 & 3,8 & 140466 & 96,2 \\
\hline La Libertad & 767 & 599632 & 20469 & 3,4 & 579163 & 96,6 \\
\hline Huancavelica & 152 & 42007 & 1105 & 2,6 & 40902 & 97,4 \\
\hline Amazonas & 276 & 91404 & 2378 & 2,6 & 89026 & 97,4 \\
\hline Lima & 3893 & 5728898 & 146911 & 2,6 & 5581987 & 97,4 \\
\hline Moquegua & 200 & 117918 & 2948 & 2,5 & 114970 & 97,5 \\
\hline Tumbes & 242 & 119467 & 2962 & 2,5 & 116505 & 97,5 \\
\hline Pasco & 129 & 40301 & 937 & 2,3 & 39364 & 97,7 \\
\hline Junín & 223 & 132638 & 2974 & 2,2 & 129664 & 97,8 \\
\hline Piura & 643 & 512861 & 10941 & 2,1 & 501920 & 97,9 \\
\hline Lambayeque & 616 & 322447 & 6694 & 2,1 & 315753 & 97,9 \\
\hline Cusco & 250 & 138323 & 2766 & 2,0 & 135557 & 98,0 \\
\hline Loreto & 421 & 205129 & 3775 & 1,8 & 201354 & 98,2 \\
\hline Ancash & 636 & 234859 & 2981 & 1,3 & 231878 & 98,7 \\
\hline Arequipa & 628 & 558534 & 6226 & 1,1 & 552308 & 98,9 \\
\hline
\end{tabular}

Ji-cuadrado $(p<0,001)$

* Muestra usada en el estudio; ** Población calculada con factores de expansión

\section{DISTRIBUCIÓN GEOGRÁFICA Y ATR}

De acuerdo con la región de procedencia, observamos que la mayor frecuencia de autorreporte de accidente de tránsito en el último año se da en la selva $(5,0 \%)$, siendo de $3,1 \%$ en la sierra y de $2,5 \%$ en la costa. Según el cálculo de la razón de probabilidades, la región de la selva presentó un OR de 2,03 (IC95\% 1,55-2,65); es decir, que los pobladores de la selva presentaron el doble de probabilidad de reportar algún accidente de tránsito con respecto a los de la región costa. Este hallazgo es estadísticamente significativo $(p<0,001)$.

Los porcentajes de autorreporte de accidente de tránsito en cada una de las regiones políticas del Perú se observan en la Tabla 2. Las cifras más altas corresponden a las regiones de Ucayali $(7,9 \%)$, Ayacucho (6,5\%), Cajamarca $(5,9 \%)$, Huánuco $(5,5 \%)$, Puno $(5,3 \%)$ y Tacna $(5,2)$. Los departamentos con menor reporte de accidente de tránsito en el último año fueron Arequipa $(1,1 \%)$, Ancash $(1,3 \%)$, Loreto $(1,8 \%)$ y Cusco $(2,0 \%)$. En el caso de Lima se estimó una prevalencia de $2,6 \%$. Igualmente,

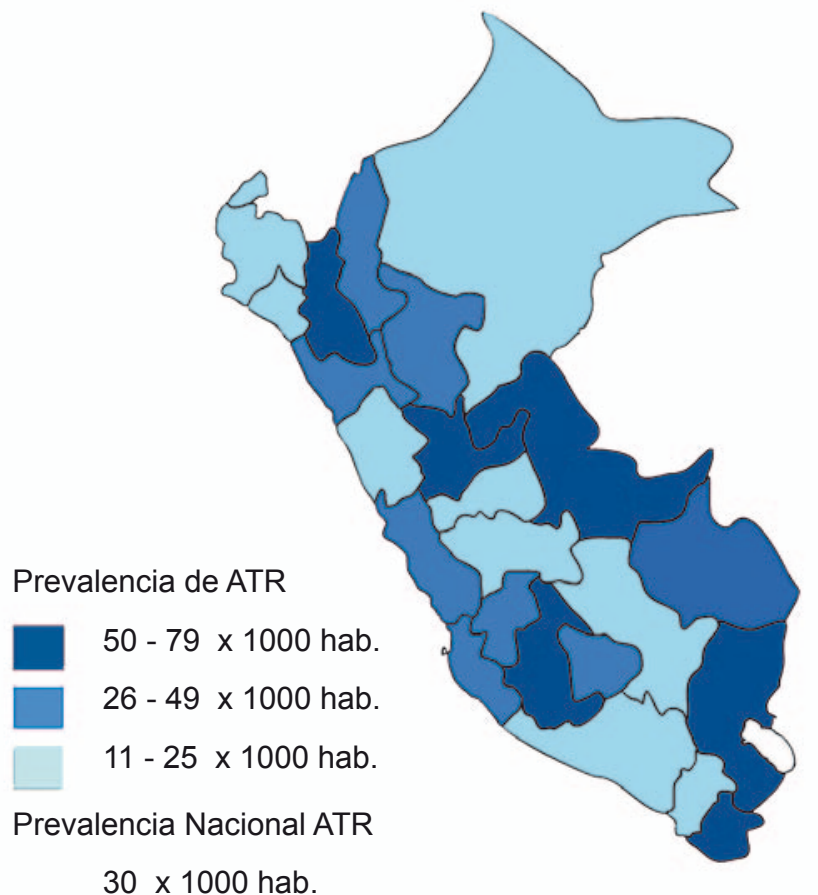

Figura 1. Distribución geográfica de la prevalencia de los accidentes de tránsito autorreportados (ATR) en el último año, Perú 2006 . 
aunque categorizados en función a la prevalencia, la Figura 1 muestra la distribución geográfica de los accidentes autorreportados. Dichas diferencias son estadísticamente significativas $(p<0,001)$.

\section{CONSUMO DE DROGAS Y ATR}

Del total de encuestados, el 67,1\% manifestó haber consumido drogas legales (alcohol o tabaco) en el último año, de los cuales, el 3,6\% reportó haber sufrido algún accidente de tránsito en el último año. Del grupo que indicó no haber consumido estas drogas el último año, el 1,9\% indicó que había tenido algún accidente de tránsito el último año. Esta diferencia resultó estadísticamente significativa $(p<0,001)$, por lo cual las personas que consumieron alguna droga legal en el último año presentó el doble de probabilidad de haber sufrido un antecedente de accidente de tránsito en el último año (OR=1,98; IC: 1,53-2,55).

Al examinar exclusivamente el consumo de alcohol en el último año y compararlo al reporte de accidente de tránsito en el último año, encontramos que el 3,7\% de los que habían consumido esta sustancia reportó haber tenido algún accidente de tránsito en el último año; mientras que el $2,0 \%$ de los que no consumieron alcohol el último año informaron haber sufrido dicho evento.

Tanto el consumo de drogas médicas como el de ilegales (marihuana, cocaína, PBC, éxtasis, crack, etc.) en el último año se asociaron con una mayor probabilidad, estadísticamente significativa, de presentar el antecedente de accidente de tránsito.

\section{DISCUSIÓN}

La información dada mediante el autorreporte en encuestas nacionales, si bien cargada de diversos sesgos, tiene valor - al ser analizada con otros indicadores - en la estimación de la verdadera magnitud de los accidentes de tránsito, brindando, sobre todo, información sobre casos leves o no fatales ${ }^{(12)}$.

La prevalencia de accidentados en el último año según el autorreporte de los encuestados en la III Encuesta Nacional de Consumo de Drogas en la Población General del Perú (DEVIDA, 2006) encontrada fue de casi 3,0\%; es decir, de 30 por cada 1000 o de 3000 por cada 100 mil habitantes a nivel nacional; esta encuesta posee representatividad nacional, por lo que estos datos son posibles de extrapolar a la población total peruana. Esta cantidad representa la gran magnitud que el problema realmente tiene en el Perú, sobre todo si lo comparamos con otras cifras publicadas o de manejo público.
En un estudio similar realizado en México por Ávila et al. (13), se encontró que la prevalencia nacional sería de 10 por 1000 habitantes; es decir, la tercera parte de lo que encontramos en el Perú en el presente estudio.

De acuerdo con los datos de la PNP, la tasa de eventos registrados por ellos sería de 289,8 x 100 mil habitantes (5); no obstante, surgen las interrogantes: ¿Cuántas personas están involucradas en estos eventos? ¿Qué sucede con los accidentes que no son informados a la PNP?; con lo encontrado en nuestro análisis, podríamos afirmar que el número de accidentes registrados por la PNP (generalmente de consecuencia fatal, con lesiones o considerable daño material) representa aproximadamente sólo el 9,6\% del total de personas accidentadas a nivel nacional de casos generalmente no fatales o leves.

Un elemento importante a tomar en cuenta para la comparación de esta información es determinar la unidad que fue sometida a análisis. El autorreporte es dado por cada persona por lo que el resultado indica el número de accidentados. Generalmente, los reportes policiales se refieren al número de accidentes (eventos), en el cual pueden estar involucradas más de una persona. Otros indicadores se basan en la cantidad de personas (lesionadas o fallecidas) con respecto al número de vehículos. El parque automotor peruano (108 vehículos por cada 100000 habitantes), si bien pequeño, resulta el más mortal de Latinoamérica, ya que se registran 300 muertes por cada 100 mil vehículos ${ }^{(4)}$. Sin embargo, resaltamos que la encuesta no recoge el dato de cantidad de accidentes por persona en el último año ni la gravedad de éstos, por lo que la cifra obtenida podría subvalorar el problema.

El hecho adquiere mayor importancia al considerar que la OMS señaló que entre los años 2000 y 2020, las muertes debidas a accidentes de tránsito presentarán una disminución cercana al $30 \%$ en países desarrollados, pero de manera contraria, aumentarán en los países de medianos y bajos ingresos como el nuestro ${ }^{(2,5)}$.

Los hallazgos con respecto al sexo y la edad de los accidentados, son compatibles con otros estudios al indicar que el sexo masculino y el grupo de edad adulto joven, son los más comprometidos ${ }^{(4,5,7,9,13)}$; explicado por ser los de mayor desplazamiento y, por consiguiente, mayor exposición ${ }^{(13)}$. Asimismo, revelan que el grado de instrucción no estaría asociado al reporte de accidente de tránsito.

Es interesante señalar que, si bien la mayor cantidad de casos está concentrada en Lima (26\%), tal como informan las fuentes oficiales, ${ }^{(4-6)}$ éstas cifras evaluadas 


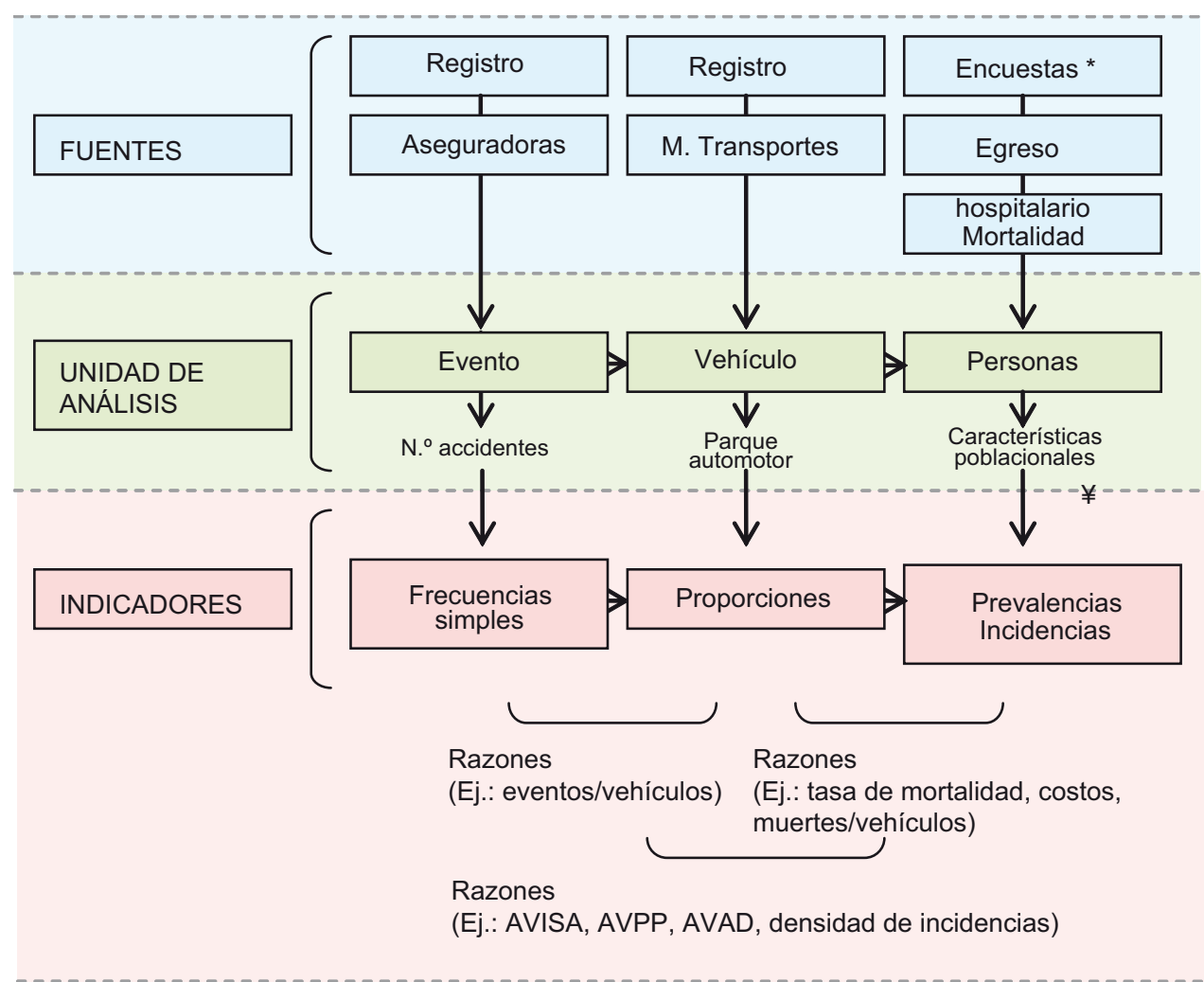

Figura 2. Sistema de información y construcción de indicadores para los accidentes de tránsito. (Propuesto por los autores)

* Encuestas nacionales en población general, como la analizada en el presente estudio

*Características socio-demográficas, sanitarias y económicas.

AVISA: años de vida saludables perdidos.

AVPP: años de vida potencialmente perdidos.

AVAD: años de vida ajustados en función de la discapacidad.

proporcionalmente nos indican que la selva es la región que presenta mayor prevalencia de accidentes no fatales (50 x 1000 hab., en promedio) siendo Ucayali, la de mayor tasa (79 x 1000 hab.), es preciso mencionar que la encuesta fue hecha principalmente en zonas urbanas del país.

En los resultados mostrados vemos cómo el consumo de drogas legales tiene una importante asociación con el autorreporte de accidente de tránsito. Estos resultados son consistentes con todos los informes e investigaciones que asocian dichos factores con los accidentes de tránsito ${ }^{(4,7,9,11,12)}$.

Es importante resaltar que existen reportes que indican que el consumo de alcohol, incluso en rangos no tóxicos, eleva el riesgo de padecer un accidente, incrementa la mortalidad y está asociado con lesiones más graves y mayores tiempos de recuperación ${ }^{(11)}$. Además, la presencia de elevadas concentraciones de alcohol en sangre de los pacientes en el momento de ocurrido un accidente de tránsito, está asociado con un posterior cambio en el diagnóstico con respecto al de la admisión a la emergencia, lo que empeora el pronóstico ${ }^{(12)}$.
En Perú se han documentado tasas altas de consumo de alcohol en conductores o peatones causantes o involucrados en accidentes de tránsito. Es aún motivo de mayor preocupación la tendencia al aumento del número de casos positivos en el dosaje etílico entre conductores en general ${ }^{(9)}$. Tras ello, es pertinente evaluar las distintas campañas de sensibilización que vienen llevando a cabo las diferentes instituciones encargadas de velar por la disminución de los accidentes de tránsito.

Luego de discutir brevemente los resultados, estimamos importante el hecho de reflexionar acerca del sistema de información sobre accidentes de tránsito en el Perú. Hasta el momento, hemos encontrado información de los registros policiales, egresos hospitalarios y personas fallecidas; lo que, a nuestro entender, cubre sólo una parte de la magnitud de los accidentes de tránsito.

En la Figura 2 se propone un modelo de los constituyentes del sistema de información sobre accidentes de tránsito que brinda la información necesaria para estimar la verdadera magnitud y velocidad de este fenómeno en la población, así como el impacto económico y en la calidad 


\begin{tabular}{|c|c|c|c|c|}
\hline \multirow{3}{*}{ EVENTO } & Registro Policial & Transporte & Atención Médica & Consecuencia \\
\hline & & & & \\
\hline & $\begin{array}{l}\text {-Total de accidentes } \\
\text {-Tipo } \\
\text {-Causa } \\
\text {-Día y hora } \\
\text {-Vehículo } \\
\text {-Avenida o vía } \\
\text {-Dosaje etílico } \\
\text {-Consecuencia } \\
\text {-Mortalidad }\end{array}$ & $\begin{array}{l}\text {-Tiempo } \\
\text {-Mortalidad }\end{array}$ & $\begin{array}{l}\text {-Sexo } \\
\text {-Edad } \\
\text {-Diagnóstico } \\
\text {-Mortalidad }\end{array}$ & $\begin{array}{l}\text {-Alta } \\
\text {-Rehabilitación } \\
\text {-Sexo } \\
\text {-Edad } \\
\text {-Diagnóstico } \\
\text {-Morgue } \\
\text {-Sexo } \\
\text {-Edad } \\
\text {-Diagnóstico } \\
\text { (Causa de Muerte) }\end{array}$ \\
\hline
\end{tabular}

Figura 3. Variables de la cobertura de un accidente de tránsito. (Modificado de Wong et al., 2009) ${ }^{(8)}$

de vida. En el esquema vemos el lugar que tienen las encuestas nacionales en población general, brindando datos que son importantes para caracterizar accidentes que incluyen un gran grupo que no es cuantificado en los registros policiales u hospitalarios.

Posteriormente, al intersectar las variables de las diferentes fuentes, podremos obtener una diversidad de razones que -vistas globalmente- nos indiquen la magnitud del problema de acuerdo a las diferentes unidades de análisis. Ahora ¿qué variables diferenciar para ser incluidas en el sistema y construir las razones? Puede que esto sea variable; como ejemplo, en un estudio realizado en el Callao ${ }^{(8)}$, se recogió información sobre los accidentes analizando datos provenientes de las instituciones que forman parte del Comité Multisectorial para la Vigilancia y Prevención de Accidentes de Tránsito del Callao construyendo un espectro de variables con el fin de caracterizar el fenómeno (Figura 3). Sin embargo, la cobertura mencionada es posterior al evento. Un enfoque integral deberá tomar en cuenta variables y situaciones previas a la ocurrencia del accidente (como las actitudes de los peatones y choferes, la educación vial, la publicidad de las bebidas alcohólicas, etc.), siempre con un fin preventivo ${ }^{(9)}$.

Finalmente, mencionamos que la información encontrada y discutida en nuestro estudio presenta algunas limitaciones. En primer lugar, al ser la unidad de análisis la persona, es difícil realizar comparaciones con otras cifras que poseen denominadores diferentes (como los obtenidos por la PNP o los publicados por el MINSA). La encuesta no recoge información de población rural ni de los diferentes estratos socioeconómicos, tampoco pregunta sobre la cantidad de accidentes sufridos por persona en el periodo estudiado ni la gravedad de éstos.

Así también, como hemos mencionado, existen sesgos importantes como el de memoria donde es posible que los encuestados no recuerden con exactitud los datos requeridos o que exista la posibilidad de respuestas fic- ticias. Es decir, constituye una evaluación subjetiva del fenómeno frente a los datos objetivos que pudiesen haber sido recabados desde el lugar mismo del accidente.

A pesar de todo ello, sostenemos que los resultados de este estudio son importantes porque nos permite una aproximación mayor a la problemática de los accidentes de tránsito en el Perú, revelando que las vallas que tenemos que superar son muy altas. Consideremos la importancia, tomando en cuenta que cada información acerca de los accidentes de tránsito resulta relevante en el afán de construir un sistema integrado de información que tenga como fin una caracterización cada vez más fidedigna del problema; paso de suma importancia para otorgar a este fenómeno -prioritaria y objetivamentelos recursos suficientes para intervenir en su pronta solución o control.

\section{Fuentes de Financiamiento}

Autofinanciado.

\section{Conflictos de Interés}

Los autores declaran no tener conflictos de interés en la publicación de este artículo.

\section{REFERENCIAS BIBLIOGRÁFICAS}

1. Economic Commission, Intersecretariat Working Group on Transport Statistics. Glossary of transport statistics, 3rd edition. Luxembourg, United Nations Economic and Social Council; 2003.

2. Organización Mundial de la Salud. Informe mundial sobre prevención de los traumatismos causados por el tránsito. Ginebra Organización Mundial de la Salud; 2004.

3. Mathers C, Roncar D. Updated projections of global mortality and burden of disease, 2002-2030: data sources, methods and results. Geneva: World Health Organization; 2005.

4. Perú, Ministerio de Salud. Plan General "Estrategia Sanitaria Nacional de Accidentes de Tránsito". Lima: MINSA, 2004 
5. Perú, Ministerio de Salud. Plan Nacional de la Estrategia Sanitaria Nacional de Accidentes de Tránsito 2009-2012. Lima: MINSA; 2008.

6. Defensoría del Pueblo, adjuntía de Medio Ambiente, Servicios Públicos y Pueblos indígenas. La Defensoría del Pueblo y el Transporte Terrestre de Pasajeros. Compendio de investigaciones defensoriales. Lima: Defensoría del Pueblo; 2010.

7. Bambarén C. Perfil de Seguridad Vial en el Perú. Lima: Ministerio de Salud; 2004.

8. Velásquez A. La carga de enfermedad y lesiones en el Perú y las prioridades del plan esencial de aseguramiento universal. Rev Peru Med Exp Salud Publica. 2009; 26(2): 222-31.

9. Wong P, Salazar D, Bérninzon L, Rodríguez A, Salazar M, Valderrama H, et al. Caracterización de los accidentes de tránsito en la región Callao-Perú, 1996-2004. Rev Peru Epidemiol. 2009; 13(3): e3

10. Alfaro-Basso D. Problemática sanitaria y social de la accidentalidad del transporte terrestre. Rev Peru Med Exp Salud Publica. 2008; 25(1): 133-7.
11. Fabbri A, Marchesini G, Morselli-Labate AM, Rossi F, Cicognani A, Dente M, et al. Positive blood alcohol concentration and road accidents. A prospective study in an Italian emergency department. Emerg Med J. 2002 May;19(3):210-4.

12. Fabbri A, Marchesini G, Morselli-Labate AM, Rossi F, Cicognani A, Dente M, et al. Blood alcohol concentration and management of road trauma patients in the emergency department. J Trauma. 2001 Mar;50(3):521-8.

13. Ávila-Burgos L, Medina-Solís CE, Pérez-Núñez R, HijarMedina M, Aracena-Genao B, Hidalgo-Solórzano E, et al. Prevalencia de accidentes de tránsito no fatales en México: resultados de la ENSANUT 2006. Salud Publica Mex. 2008; 50 (suppl 1): S38-S47.

14. Comisión Nacional para el Desarrollo y Vida Sin Drogas (DEVIDA). III Encuesta Nacional de Consumo de Drogas en la Población General del Perú 2006. Lima: DEVIDA; 2007.

Correspondencia: Paolo Wong Chero

Dirección: Av. Paseo de la República 7750-E301, Los Rosales, Lima 33, Perú.

Correo electrónico: pwongc@epiredperu.net

Consulte las ediciones anteriores de la Revista Peruana de Medicina Experimental y Salud Pública en

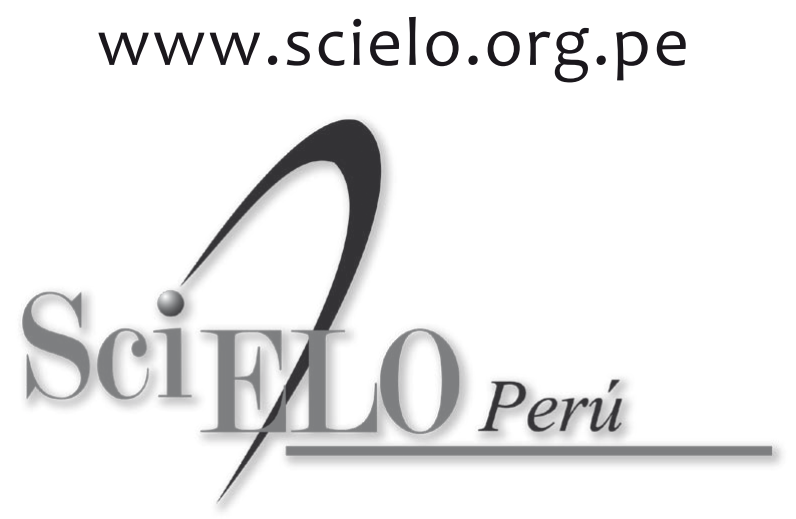

